\title{
Transverse beam coupling impedance of the CERN Proton Synchrotron
}

\author{
S. Persichelli* and M. Migliorati \\ University of Rome 'La Sapienza', Via A. Scarpa 14, 00161 Roma, Italy \\ N. Biancacci, S. Gilardoni, E. Metral, and B. Salvant \\ CERN, CH-1211, Geneva 23, Switzerland \\ (Received 7 January 2016; published 8 April 2016)
}

\begin{abstract}
Beam coupling impedance is a fundamental parameter to characterize the electromagnetic interaction of a particle beam with the surrounding environment. Synchrotron machine performances are critically affected by instabilities and collective effects triggered by beam coupling impedance. In particular, transverse beam coupling impedance is expected to impact beam dynamics of the CERN Proton Synchrotron (PS), since a significant increase in beam intensity is foreseen within the framework of the LHC Injectors Upgrade (LIU) project. In this paper we describe the study of the transverse beam coupling impedance of the PS, taking into account the main sources of geometrical impedance and the contribution of indirect space charge at different energies. The total machine impedance budget, determined from beam-based dedicated machine measurement sessions, is also discussed and compared with the theoretical model.
\end{abstract}

DOI: 10.1103/PhysRevAccelBeams.19.041001

\section{INTRODUCTION}

The goal of the LHC injectors upgrade (LIU) project [1], started in 2010, is to provide reliable beams with the challenging characteristics required by the High Luminosity LHC [2] program, until at least 2030. The final objective of the upgrade is to reduce the statistical error in the measurements at the interaction points of LHC. For the future operation at higher intensities and beam brightness required by the LIU project, it is of fundamental importance to detect the main sources of wakefields and estimate with precision the coupling impedance of all the machines in the LHC injection chain, in order to minimize collective effects triggered by the coupling impedance and improve quality and brightness of the colliding beams. The study of the PS longitudinal broadband impedance was addressed for the first time in the late 1970s, where a measurements campaign, based on longitudinal stability during debunching and on quadrupole beam transfer functions, showed a longitudinal impedance of about $25 \Omega$ at extraction energy [3]. Since important hardware changes and new devices installations were foreseen during the LHC long Shutdown (LS1) [4], a similar study has been carried out again in 2012 [5]. The study revealed a broadband longitudinal impedance of about $18 \Omega$.

*serena.persichelli@uniroma1.it

Published by the American Physical Society under the terms of the Creative Commons Attribution 3.0 License. Further distribution of this work must maintain attribution to the author(s) and the published article's title, journal citation, and DOI.
In this paper we present the study of the PS transverse beam coupling impedance. We computed the horizontal and vertical beam coupling impedance model of the machine, with the help of beam based measurements and numerical codes. This study, together with the previous knowledge of the PS longitudinal broadband impedance model, allows us to better understand instability thresholds [6], to predict the effects of the beam intensity increase planned for the upgrade program, and in general to account for the impact of all hardware changes in the PS foreseen for the next years.

The paper is organized as follows: in Sec. II we describe the method we have used to determine the imaginary part of the total transverse effective impedance of the machine from betatron tune measurements, while in Sec. III we discuss the measurement results at different energies. In Sec. IV analytical transverse impedance calculations of the devices installed in the machine predicted to be important sources of impedance, including the indirect space charge contribution, are presented. In Sec. V we discuss the impedance localization method that we have used to check our impedance model and in Sec. VI we compare the results of measurements and simulations. In Sec. VII we discuss the impact of the chromaticity on the tune shift measurements and impedance evaluation.

\section{TUNE SHIFT MEASUREMENTS FOR TRANSVERSE IMPEDANCE DETERMINATION}

\section{A. Tune shift theory and measurements}

Measuring the betatron coherent frequency shift with beam intensity gives information on the total reactive transverse impedance according to Sacherer's theory 
[7,8]. For a Gaussian bunch of r.m.s. bunch length $\sigma_{z}$ traveling with velocity $v=\beta c$, the coherent tune shift $\Delta Q$ is proportional to the imaginary part of the transverse effective impedance $Z_{t}^{\text {eff }}$ by [9]

$$
\Delta Q=-\frac{\beta e I_{0}}{4 \sigma_{z} \sqrt{\pi} \omega_{0}^{2} \gamma Q_{0} m_{0}} \operatorname{Im}\left[Z_{t}^{\mathrm{eff}}\right],
$$

where $I_{0}$ is the bunch current, $Q_{0}$ is the unperturbed betatron tune, $\gamma$ is the relativistic factor, $e$ the particle charge, $\omega_{0}$ the angular revolution frequency and $m_{0}$ the particle mass at rest. If the bunch length is kept constant during the measurement, Eq. (1) predicts a tune shift linear with bunch current, with a slope proportional to the imaginary part of the transverse total effective impedance. If we assume a Gaussian zero azimuthal bunch mode, with power spectrum given by

$$
h(\omega)=e^{-\left(\omega^{2} \sigma_{z}^{2} / c^{2}\right)},
$$

the effective transverse impedance is defined as the impedance weighted by the transverse bunch spectrum centered at the chromatic frequency $\omega_{\xi}$ :

$$
Z_{t}^{\mathrm{eff}}=\frac{\sum_{p=-\infty}^{\infty} Z_{t}\left(\omega^{\prime}\right) h\left(\omega^{\prime}-\omega_{\xi}\right)}{\sum_{p=-\infty}^{\infty} h\left(\omega^{\prime}-\omega_{\xi}\right)},
$$

where $\omega^{\prime}=\omega_{0}\left(p+Q_{0}\right)$ (with $p$ an integer). The chromatic frequency is defined as

$$
\omega_{\xi}=\frac{\omega_{0} Q_{0} \xi}{\eta}
$$

where $\eta$ is the slip factor and $\xi$ is the chromaticity defined here as the betatron tune variation with the momentum:

$$
\xi=\frac{\Delta Q / Q_{0}}{\Delta p / p_{0}} .
$$

To perform betatron tune measurements of a single bunch beam in the PS, we first need to excite the betatron oscillation with a chirp signal produced by a kicker. The transverse tunes $Q_{x}$ and $Q_{y}$ are then measured with a system based on diode detection, known as base band tune (BBQ) [10]. The transverse position of the bunch center of mass is acquired every turn by a beam position monitor (BPM) located in straight section 72, a cylindrical pickup provided by four stripline electrodes. High amplitude short pulses obtained from the BPM electrodes are sent to a diode detector, which converts the modulation of the pulses, related to beam oscillations, into a signal in the audio frequency range. The betatron tune is then obtained from a Fast Fourier Transform (FFT) of this signal, and corresponds to the maximum peak value.

\section{B. Control of working point}

Bending and focusing of the beam is provided by the main magnet units of the PS; the working point, in the absence of any auxiliary magnets or coils, is determined by the momentum of the beam. For example, the bare machine at the energy of $2 \mathrm{GeV}$ is working in linear condition (natural working point) with measured tunes of $Q_{x}=6.253$, $Q_{y}=6.285$, and chromaticities of $\xi_{x}=-0.83, \xi_{y}=-1.12$, corresponding to chromatic angular frequencies of $213 \mathrm{MHz}$ and $280 \mathrm{MHz}$, respectively. To correct the effects of linear and second order chromaticity in the PS, the magnetic field higher order components are controlled with auxiliary windings, called pole face windings (PFW) [11]. The PS PFW, consisting of four coils mounted on the iron poles of each magnet (two coils for the focusing and two for the defocusing yoke), are used to control the working point and chromaticity. An additional coil, called figure-of-eight loop, creates opposite fields in the two magnet yokes. Using the combination of the PFW and the figure-of-eight loop, the horizontal and vertical tunes, horizontal and vertical chromaticities, and another parameter (for example the nonlinear chromaticity), can be controlled by five parameters independently.

\section{TUNE SHIFT MEASUREMENTS AT ZERO CHROMATICITY}

To better understand the contribution of indirect space charge to the total impedance budget, four different energy values have been considered for tune shift measurements. In Table I the main parameters of the beams used in the four cases are summarized. Tune shift measurements have been performed during several machine development (MD) sessions on a dedicated cycle [12]. The magnetic field as a function of time of the cycle used for measurements at $25.48 \mathrm{GeV}$ is shown in Fig. 1. Tune shift measurements are acquired only on the flat top of the magnetic field at the constant kinetic energy of $25.48 \mathrm{GeV}$.

The vertical tune shift $\Delta Q_{y}$ is more significant and easy to observe than the horizontal tune shift $\Delta Q_{x}$, since many devices in the PS circumference have a large aspect ratio and can be approximated with a parallel plates geometry (i.e., kicker magnets). If we approximate the geometry of the PS vacuum chamber with two conducting parallel plates, the dipolar and quadrupolar components of the impedance [13] exhibit the same sign in the vertical plane. Instead, in the horizontal plane, the parallel plates model exhibits zero total impedance due to the perfect compensation of dipolar and quadrupolar components of the

TABLE I. Beam parameters for vertical tune shift measurements at different energies.

\begin{tabular}{lcccc}
\hline \hline $\mathrm{E}_{\mathrm{kin}}[\mathrm{GeV}]$ & 25.48 & 13.09 & 7.25 & 2 \\
\hline$\gamma_{\text {rel }}$ & 27.77 & 14.95 & 8.72 & 3.13 \\
Slip factor $\eta$ & 0.052 & 0.022 & -0.013 & -0.075 \\
Harmonic number & 8 & 8 & 8 & 16 \\
$4 \sigma_{\tau}[\mathrm{ns}]$ & 45 & 55 & 55 & 80 \\
\hline \hline
\end{tabular}




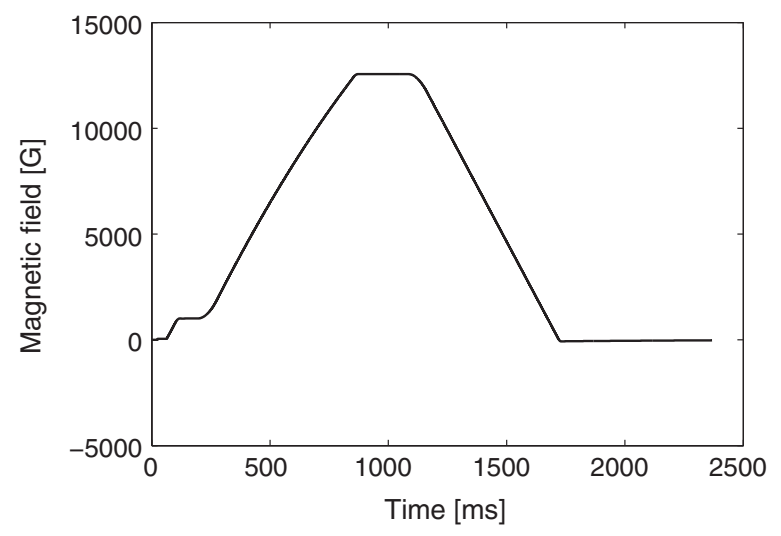

FIG. 1. Magnetic field as a function of time of the $25.48 \mathrm{GeV}$ beam cycle.

impedance [14]. It is then very difficult to estimate the effective horizontal impedance from the slope of a tune shift measurement. An example of a horizontal tune shift at the energy of $25.48 \mathrm{GeV}$ is shown in Fig. 2. The computed horizontal effective impedance is $0.08 \pm 0.05 \mathrm{M} \Omega / \mathrm{m}$.

The measured vertical tune shifts with beam intensity at different energies are shown in Fig. 3. Several tunes have been acquired for each energy and intensity value. The dots represent the mean value of the acquired tunes for a given intensity, while the error bars represent the standard deviation of the measured tunes. With a plain FFT, the uncertainty of the single measurements would be given by $1 / N_{\text {turns }}$. However, since the BBQ performs an interpolated FFT, the uncertainties are lower than $1 / N_{\text {turns }}$, and are in the shadow of the machine reproducibility, which defines the measurement uncertainties. We processed 2048 turns at 2 and $13.09 \mathrm{GeV}, 1024$ turns at $7.25 \mathrm{GeV}$ and 4096 at $25.48 \mathrm{GeV}$. For each plot, the vertical chromaticity has been set to a value as close as possible to zero on the PS working point. The tune is decreasing when the beam intensity increases in the bunch: according to Eq. (1), the effective impedance (including the indirect space charge) introduces a defocusing effect. On the vertical plane, both the dipolar

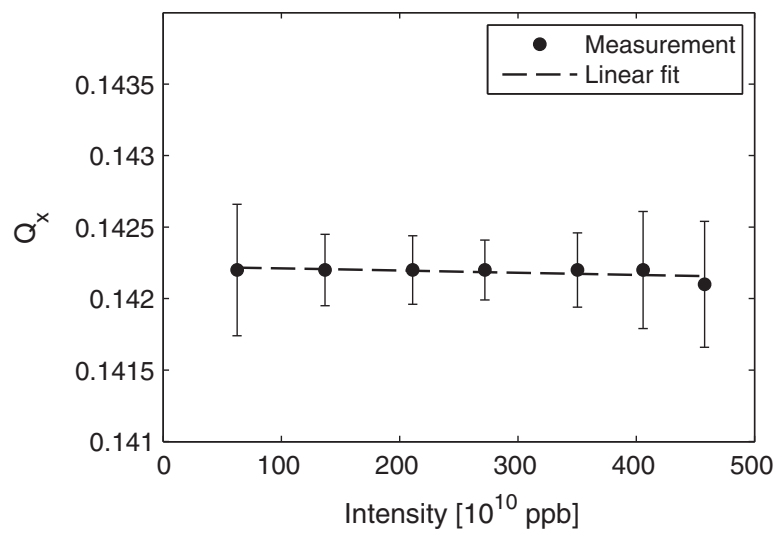

FIG. 2. Horizontal tune shifts with beam intensity measured at $25.48 \mathrm{GeV}$. and quadrupolar impedance components contribute to the tune shift. All the tune shift measurements are acquired on the flat top of the magnetic field, using dedicated magnetic cycles. The three sets of measurement at 25.48, 13.09 and $7.25 \mathrm{GeV}$ were performed injecting a TOF-type beam in the PS from the PS Booster. TOF is a beam dedicated to the n-TOF (neutron-Time Of Flight) facility, with very high nominal beam intensity $\left(7.5 \times 10^{12} \mathrm{ppb}\right)$. This type of beam allows the study with a very broad range of intensity, and a good harmonic tune signal. Only for the tune shift measurement performed at $2 \mathrm{GeV}$, an LHCINDIV (LHC individual bunch physics) beam was injected in the PS from the PS Booster. This beam is required in the commissioning filling patterns and MD for the LHC, and allows smaller intensity ranges with respect to TOF. The use of LHCINDIV drastically reduced the beam intensity range (between 15 and $35 \times 10^{10} \mathrm{ppb}$ ), but allowed to work with a smaller beam emittance and a cleaner, very peaked tune signal, decreasing the error in the tune evaluation. Some examples of typical TOF and LHCINDIV beam spectra can be found in [12].

Since at $2 \mathrm{GeV}$ the PS machine is coupled in the horizontal and vertical planes, an adjustment of the current in the skew quadrupole was needed in order to correctly perform the measurement. The current was changed from the default value (used for the other three sets of measurement at higher energies) of $0.35 \mathrm{~A}$ and $-0.35 \mathrm{~A}$ [15] to an optimum value of $0.39 \mathrm{~A}$ and $-0.39 \mathrm{~A}$, allowing to decrease the coupling between the two planes and increase the accuracy of the vertical tune measurement. Bunch lengths have been monitored and measured during all the tune shift acquisition with a dedicated tool. For each energy considered, we measured a stable bunch length at different intensities, and therefore we assumed it constant during the intensity scan for each set of tune shift measurement. In Table II the imaginary parts of the vertical effective impedance measured at different energies and zero chromaticity, assuming a constant bunch length and vertical chromaticity as close as possible to zero, are summarized. These values represent the vertical impedance budget of the PS and will be directly compared with the theoretical impedance model discussed in Sec. IV.

\section{TRANSVERSE IMPEDANCE MODEL FROM SIMULATIONS}

We computed the total transverse impedance as a function of frequency with the help of numerical codes and analytical expressions [16-18]. We calculated the individual contribution of each machine element, summing together the total transverse impedances as a function of frequency. The total impedance is always considered as the sum of the dipolar and the quadrupolar component of the impedance. The imaginary part of the effective impedance has been obtained using Eq. (3). This method allows us to monitor and compare the numerical and analytical 

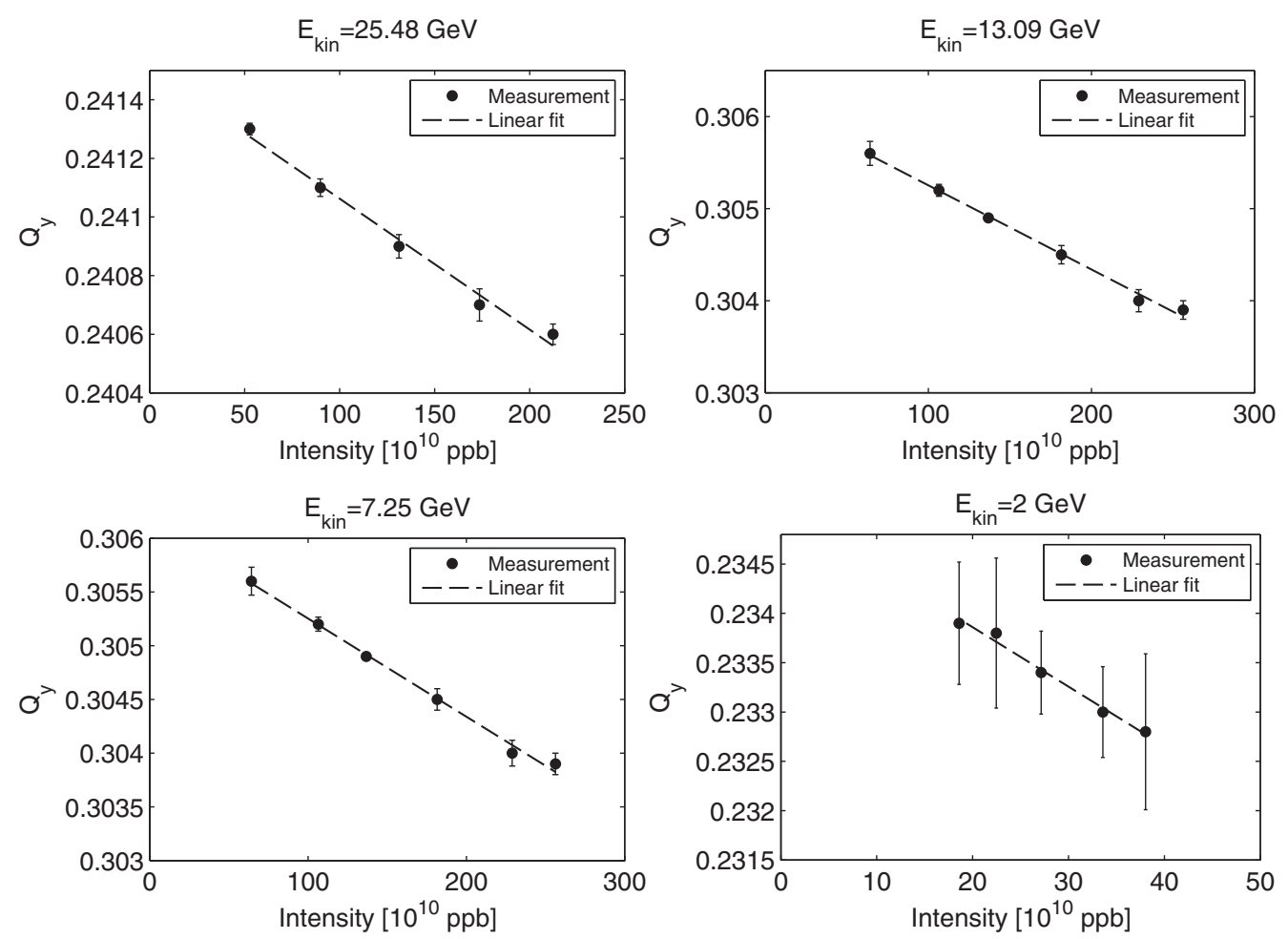

FIG. 3. Vertical tune shifts with beam intensity measured at different energies and the vertical chromaticity set to a value as close as possible to zero.

evaluation with the results of the measurements at different energies summarized in Table II.

\section{A. Indirect space charge and resistive wall}

The observed difference in the effective impedance in the vertical plane between tune shift measurements at different energies summarized in Table II can be explained by the effect of the coherent indirect space charge, induced by a beam traveling inside a perfectly conducting infinitely smooth beam pipe. To assess the contribution of indirect space charge on the impedance budget, calculations were performed with the code described in [19] for each energy considered in beam based measurements. The code allows us to describe the beam pipe geometry as a round chamber or as parallel plates. The output of the code provides a transverse impedance, as a function of frequency, that includes the contribution of space charge and resistive wall summed together for the entire machine length. Since

TABLE II. Imaginary part of the vertical effective impedance measured at different energies and zero chromaticity.

\begin{tabular}{lcc}
\hline \hline $\mathrm{E}_{\text {kin }}[\mathrm{GeV}]$ & $Z_{y}^{\text {eff }}[\mathrm{M} \Omega / \mathrm{m}]$ & $4 \sigma_{\tau}[\mathrm{ns}]$ \\
\hline 25.48 & $2.23 \pm 0.05$ & 45 \\
13.09 & $3.06 \pm 0.12$ & 55 \\
7.25 & $3.51 \pm 0.13$ & 55 \\
2 & $5.50 \pm 0.38$ & 80 \\
\hline \hline
\end{tabular}

the main PS beam chamber has an elliptical cross-section of vertical half-aperture of $35 \mathrm{~mm}$ and horizontal half-aperture of $73 \mathrm{~mm}$, a round chamber of $35 \mathrm{~mm}$ radius has been taken into account as a first approximation. An equivalent radius of $39 \mathrm{~mm}$ calculated according to [20], was also considered for comparison.

We assumed a machine made of stainless steel [5] 316LN (about $70 \%$ of the total length) with resistivity of $75 \times 10^{-08} \Omega \mathrm{m}$, and of Inconel X750 alloy (about 20\%) with resistivity of $120 \times 10^{-08} \Omega \mathrm{m}$. Approximating the PS elliptic vacuum chamber with a round geometry of radius $35 \mathrm{~mm}$, the contribution to the imaginary part of the total effective vertical impedance is $3.3 \mathrm{M} \Omega / \mathrm{m}$ at $2 \mathrm{GeV}$ and decreases drastically to $0.22 \mathrm{M} \Omega / \mathrm{m}$ at $25.48 \mathrm{GeV}$. If we consider the equivalent radius of $39 \mathrm{~mm}$, the contribution is $2.58 \mathrm{M} \Omega / \mathrm{m}$ at $2 \mathrm{GeV}$ and $0.16 \mathrm{M} \Omega / \mathrm{m}$ at $25.48 \mathrm{GeV}$. In Table III the results of the indirect space charge and

TABLE III. Indirect space charge and resistive wall vertical effective impedance expressed in $\mathrm{M} \Omega / \mathrm{m}$, calculated for different kinetic energies for a round chamber of $35 \mathrm{~mm}$ radius and $39 \mathrm{~mm}$ radius.

\begin{tabular}{lcc}
\hline \hline $\mathrm{E}_{\text {kin }}[\mathrm{GeV}]$ & $35 \mathrm{~mm}$ & $39 \mathrm{~mm}$ \\
\hline 2 & 3.30 & 2.58 \\
7.25 & 0.59 & 0.46 \\
13.09 & 0.33 & 0.25 \\
25.48 & 0.22 & 0.16 \\
\hline \hline
\end{tabular}


resistive wall contributions are summarized for each energy and the two pipe radius.

If we assume a round chamber with $35 \mathrm{~mm}$ radius, the computed indirect space charge and resistive wall contributions explain $60 \%$ of the vertical effective impedance measured at $2 \mathrm{GeV}$, and about $10 \%$ of the effective impedance measured at $25.48 \mathrm{GeV}$. Moreover, if we subtract the space charge and resistive wall contribution to the measured impedance budget summarized in Table II, we can observe that the effective impedance generated by the geometrical impedance in the machine, which should not depend on energy, has a value between 2 and 2.9 $\mathrm{M} \Omega / \mathrm{m}$. In the following section we evaluate the contribution of the most important machine elements to the total geometrical impedance.

\section{B. Kicker magnets}

Kicker magnets are generally considered a significant source of transverse impedance in synchrotron machines. To investigate the behaviour of the beam coupling impedance with respect to frequency, CST Particle Studio [16] and the Tsutsui theoretical formula [21], are considered very reliable tools to be applied for different type of kickers geometries.

A combination of six fast kickers and five lumped magnets are used in the PS for beam injection and extraction using several techniques.

Fast kickers in the PS are ferrite-loaded delay line magnets, with a rectangular-shaped aperture and ferrite split longitudinally in several cells. In each module there are two conducting cables, that are independently grounded or connected to the high voltage. To compute the transverse impedance for these devices, we imported a full mechanical 3D model from CATIA [22] into CST Particle Studio. The model has been cleaned from all the unnecessary details in order to reduce the simulation time and improve the discretization. An example of a simplified model of a ferrite-loaded delay line kicker used for CST simulations is shown in Fig. 4.

The lumped magnets are used for continuous transfer (CT) extraction and ions injection; the ferrite is not split longitudinally but a brick of aluminium is inserted in the yoke in order to reduce the impedance seen by the beam. Since 3D mechanical models were not available for this type of magnet, the model for CST simulations has been built considering the original drawings of the devices. Simulations showed that the presence of the metallic insert introduces an asymmetric perturbation in the electromagnetic field acting on the beam. This field provokes a strong monopolar component of the transverse impedance, that makes the computation of the transverse impedance inaccurate. For this reason we consider in the total machine impedance calculation the contribution of the five PS lumped kickers without the metallic insert. An example of two lumped kickers models (with the metallic insert)

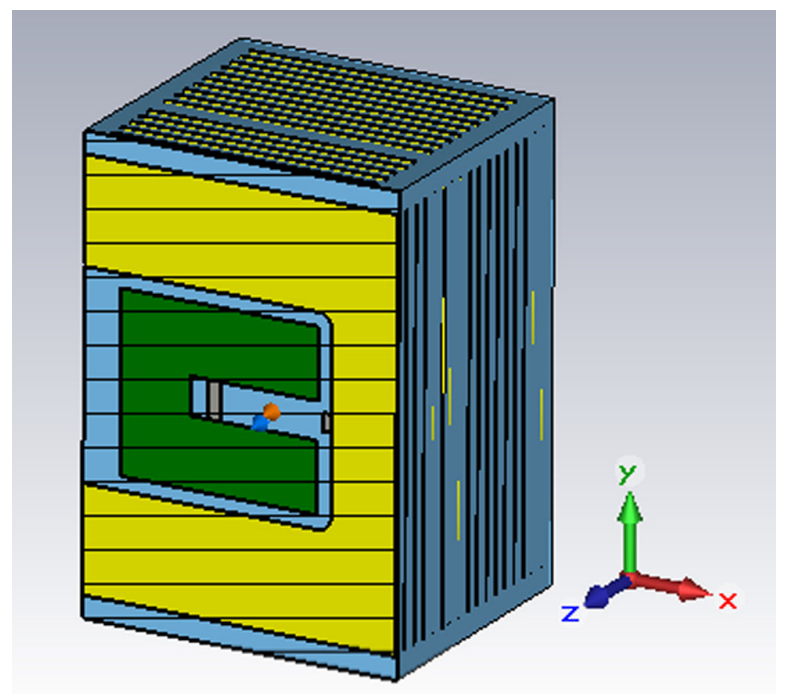

FIG. 4. Transverse section view of a ferrite-loaded delay line kicker model used for CST simulations of KFA13. The kicker is made of three identical modules. In each module the ferrite (in green) is split longitudinally in nine cells. Each cell is $24 \mathrm{~mm}$ long: $19 \mathrm{~mm}$ of ferrite and $5 \mathrm{~mm}$ of aluminium (in yellow). In each module there are two conducting cables, modeled in CST with PEC (perfectly conducting boundaries, in grey). Vacuum is represented in light blue. This model has been discretized in CST with $7,588,152$ mesh cells and simulated with a wake length of $25 \mathrm{~m}$.

implemented in CST is shown in Fig. 5. As mentioned, to calculate the kickers transverse impedance, we can apply the Tsutsui formula, that allows to compute the transverse impedance considering as input only the vertical and horizontal semiaperture of the magnet, the width of the ferrite and the total length of the ferrite. Even if this method, in general, provides very good and reliable results at high frequencies, due to the presence of segmentation and of the metallic inserts, it seems to not evaluate correctly the low frequency impedance of the PS kickers. Nevertheless this method has been extensively used for the PS kickers to cross-check results from simulations, showing a very good agreement in the range of frequency 1-10 GHz. The kickers geometrical parameters considered for Tsutsui computation are summarized in Table IV. Kickers named with the prefix KFA are lumped or delay line magnets designed with two conducting cables, while kickers named with the prefix BFA are lumped magnets designed with four conducting cables (suffix P/S refers to the pedestal and staircase module). The number following the prefix refers to the straight section number where the kicker is installed. The transverse impedance of all the PS kickers listed in Table IV has been computed with CST Particle Studio. Each kicker was analyzed with dedicated simulations, taking into account the different geometries and configurations of the ferrite cells inside the tank. For example, even if KFA79 and KFA13 are realized with the same number of modules, made by ferrite cells of equal 

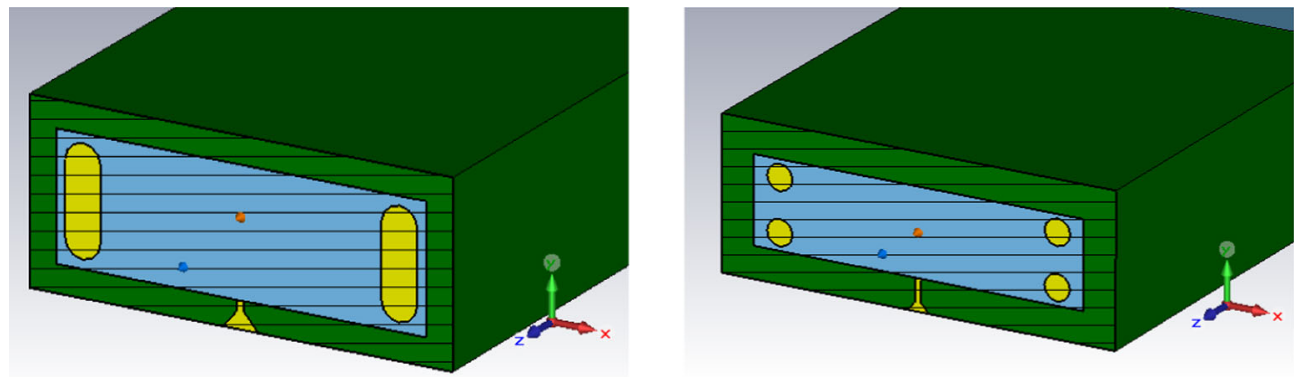

FIG. 5. Transverse section view of lumped kicker models used for CST simulations of PS kicker KFA28 (left) and BFA09S-21S (right). The kickers are made of a single ferrite yoke (in green). A brick of aluminium is inserted in the yoke in order to reduce the impedance seen by the beam. Kickers with the prefix KFA are designed with two conducting cables, while kickers with the prefix BFA are designed with four conducting cables, modeled in CST as aluminium (in yellow). Vacuum is represented in light blue.

dimensions, in KFA79 the three modules have a $180^{\circ}$ rotation from one to the next. Kicker KFA71 was built with nine modules with the same configuration of KFA79, and it is the longest kicker installed in the PS. Kicker BFA09P21P and BFA09S-21S were built with the same module geometry, but with different dimension and shapes of the conducting cables. Each kicker was discretized in CST with hexahedral mesh cells. The number of mesh cells depends, in general, on the size of the object and on the bunch length adopted. A bunch length of $10 \mathrm{~mm}$ has been chosen, in order to compute the impedance model in the range $0-10 \mathrm{GHz}$. We adopted a wake length in the range 10-20 m, depending on the length of the kicker.

For each kicker we performed four different simulations to compute the dipolar and quadrupolar component of the impedance along the two axis. For dipolar impedance the simulation was performed placing the wake integration in the center of the kicker, with the source beam displaced along $x / y$. For quadrupolar impedance the simulation was performed placing the source beam in the center of the kicker, and the integration path displaced along $x / y$. The

TABLE IV. PS kickers geometrical parameters: according with Tsutsui's nomenclature, a is the horizontal semiaperture of the magnet (between the two conductors), b is the vertical semiaperture of the magnet, $h$ is the width of the ferrite and $\mathrm{L}$ is the total length of the ferrite (consider from the first cell to the last cell for delay line magnets).

\begin{tabular}{lcccc}
\hline \hline & $\mathrm{a}[\mathrm{mm}]$ & $\mathrm{b}[\mathrm{mm}]$ & $\mathrm{h}[\mathrm{mm}]$ & $\mathrm{L}[\mathrm{m}]$ \\
\hline KFA04 & 56.5 & 37 & 40 & 0.570 \\
KFA13 & 73.5 & 26.7 & 100 & 0.730 \\
KFA21 & 73.5 & 26.7 & 100 & 0.730 \\
KFA45 & 76 & 26.6 & 40 & 0.957 \\
KFA71 & 73.5 & 26.7 & 100 & 2.190 \\
KFA79 & 73.5 & 26.7 & 100 & 0.730 \\
KFA28 & 78.5 & 35 & 15 & 0.900 \\
BFA09S & 79 & 26.25 & 20 & 0.540 \\
BFA21S & 79 & 26.25 & 20 & 0.540 \\
BFA09P & 79 & 26.25 & 20 & 0.540 \\
BFA21P & 79 & 26.25 & 20 & 0.540 \\
\hline \hline
\end{tabular}

total horizontal impedance and the total vertical impedance of the PS kickers as a function of frequency, obtained by summing all the real and imaginary parts of the transverse impedances (dipolar and quadrupolar components) computed with CST, and weighted by the $\beta$ function defined in the $2 \mathrm{GeV}$ lattice, are shown in Fig. 6 . The sign of the impedance is due to the CST notation. The real part of the
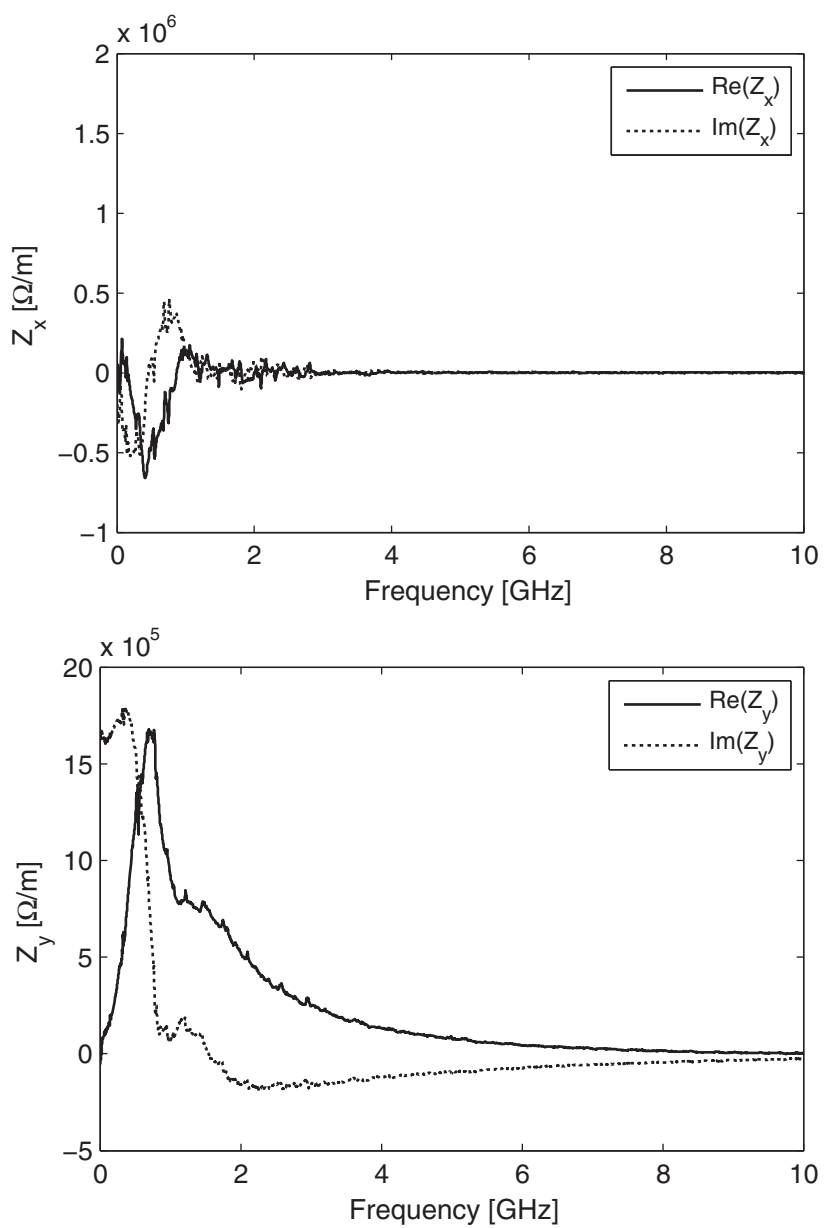

FIG. 6. Total horizontal and vertical impedance of the PS kickers (sum of dipolar and quadrupolar components), weighted for the $\beta$-function, computed with CST Particle Studio. 
TABLE V. PS Kicker imaginary parts of effective horizontal and vertical impedances weighted by the $\beta$ function defined in the $2 \mathrm{GeV}$ lattice.

\begin{tabular}{lcccc}
\hline \hline & $Z_{x}^{\text {eff }}[\Omega / \mathrm{m}]$ & $Z_{y}^{\text {eff }}[\Omega / \mathrm{m}]$ & $\beta_{x}$ & $\beta_{y}$ \\
\hline PI.KFA04 & $7.40 \mathrm{e} 03$ & $8.0 \mathrm{e} 04$ & 12.11 & 22.46 \\
PE.KFA13 & $-4.15 \mathrm{e} 04$ & $1.05 \mathrm{e} 05$ & 20.68 & 12.53 \\
PE.KFA21 & $-3.80 \mathrm{e} 04$ & $1.05 \mathrm{e} 05$ & 18.82 & 12.80 \\
PI.KFA45 & $-4.01 \mathrm{e} 04$ & $1.23 \mathrm{e} 05$ & 22.99 & 11.60 \\
PE.KFA71 & $-1.60 \mathrm{e} 05$ & $3.16 \mathrm{e} 05$ & 19.17 & 12.75 \\
PE.KFA79 & $-6.43 \mathrm{e} 04$ & $1.04 \mathrm{e} 05$ & 23.59 & 12.56 \\
PI.KFA28 & $1.27 \mathrm{e} 04$ & $1.22 \mathrm{e} 05$ & 14.64 & 20.33 \\
PE.BFA09S & $2.17 \mathrm{e} 04$ & $8.28 \mathrm{e} 04$ & 22.84 & 13.13 \\
PE.BFA21S & $1.86 \mathrm{e} 04$ & $8.00 \mathrm{e} 04$ & 19.27 & 12.74 \\
PE.BFA09P & $1.59 \mathrm{e} 04$ & $8.31 \mathrm{e} 04$ & 22.84 & 13.13 \\
PE.BFA21P & $1.86 \mathrm{e} 04$ & $8.03 \mathrm{e} 04$ & 19.27 & 12.74 \\
\hline \hline
\end{tabular}

vertical impedance shows a peak around $700 \mathrm{MHz}$ that is consistent with the frequency of the instability at transition observed in [23]. The imaginary part of the vertical impedance increases for frequencies lower than $700 \mathrm{MHz}$, and drastically drop at the resonance. This behavior partially explains the measurements that are going to be presented in Sec. VII. The kicker contribution to the imaginary part of the vertical effective impedance of the PS is about $1.3 \mathrm{M} \Omega / \mathrm{m}$, while the horizontal contribution is almost zero due to the compensation of dipolar and quadrupolar components of the impedance, as predicted in Sec. III. Kickers magnets can explain about $25 \%$ of the vertical effective impedance measured at $2 \mathrm{GeV}$, and about $60 \%$ of the vertical effective impedance measured at $25.48 \mathrm{GeV}$. The imaginary parts of the total effective horizontal and vertical impedances introduced by each kicker (sum of dipolar and quadrupolar components), weighted by the $\beta$ function defined in the $2 \mathrm{GeV}$ lattice, are summarized in Table V. It is worth noting that the most significant contribution is due to the kicker located in straight section 71 (KFA71): the vertical effective impedance increases for kicker with the total length of the ferrite. In Sec. V we are going to show that KFA71 can be identified as one of the major source of transverse geometrical impedance applying the impedance localization measurement technique.

\section{C. rf cavities}

The contribution of the $10,40,80$, and the $200 \mathrm{MHz}$ cavities, weighted by the $\beta$ function defined in the lattice, has been included in the PS transverse impedance model. Simplified models of the 40 and $80 \mathrm{MHz}$ cavity were first implemented with the 2D code ABCI [17], starting from the design described in [24]. At a later stage, 3D models have been realized in CST Particle Studio. The models do not include couplers and tuning system of the cavities. Results from CST and ABCI simulations were in very good agreement, showing that rf cavities have a negligible impact on the transverse impedance budget. Indeed, their contribution explains less than $1 \%$ of the of the vertical effective impedance measured at different energies.

\section{Vacuum equipment}

Vacuum equipment and devices like pumps, sector valves, bellows, steps, and metallic flanges were included in the transverse impedance model. All the mentioned devices were simulated with CST Particle Studio. In Fig. 7, 3D models of a PS flange and a sector valve used for simulations with CST, are shown. In Fig. 8, 3D models of a PS vacuum pump and a bellow used for simulations with CST, are shown. Since the vacuum equipments, in general, are not localized in a single straight section but occur in many locations inside the machine, they have been treated in the computation as distributed elements and weighted by the averaged $\beta$ function defined in the lattice. The elements included in the theoretical impedance model and their effective horizontal and vertical impedances, computed with CST Particle studio and scaled by the total number of elements, are listed in Table VI.

Even if the impedance of the single element is predicted to be negligible, the sum of many distributed elements can become significant. Vacuum pumps and bellows, due to the large number of elements installed in the machine, are two of the major sources of impedance in the machine.
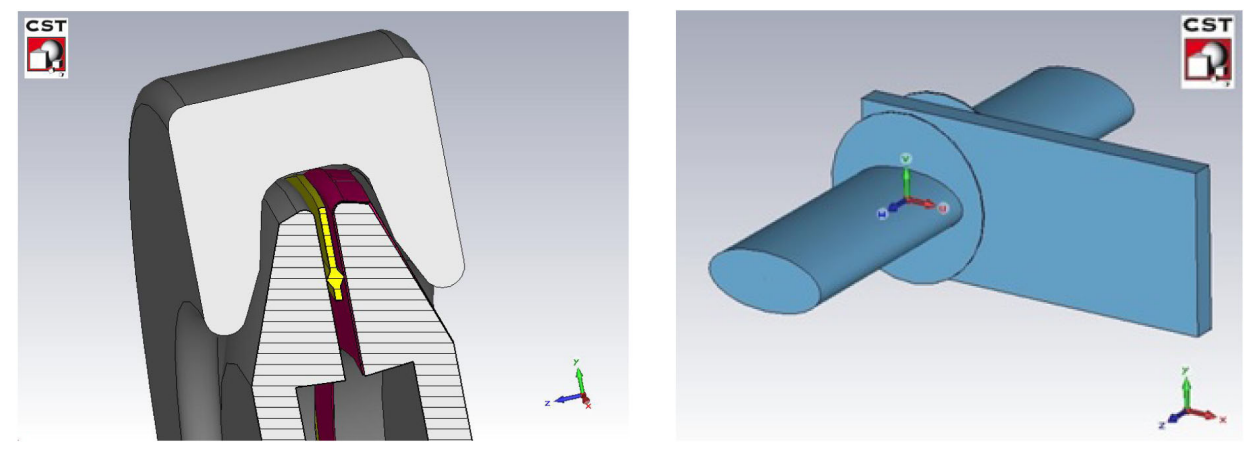

FIG. 7. 3D model of a PS flange used for CST Particle Studio simulations (left); 3D model of a PS sector valve used for CST Particle Studio simulations (right). 

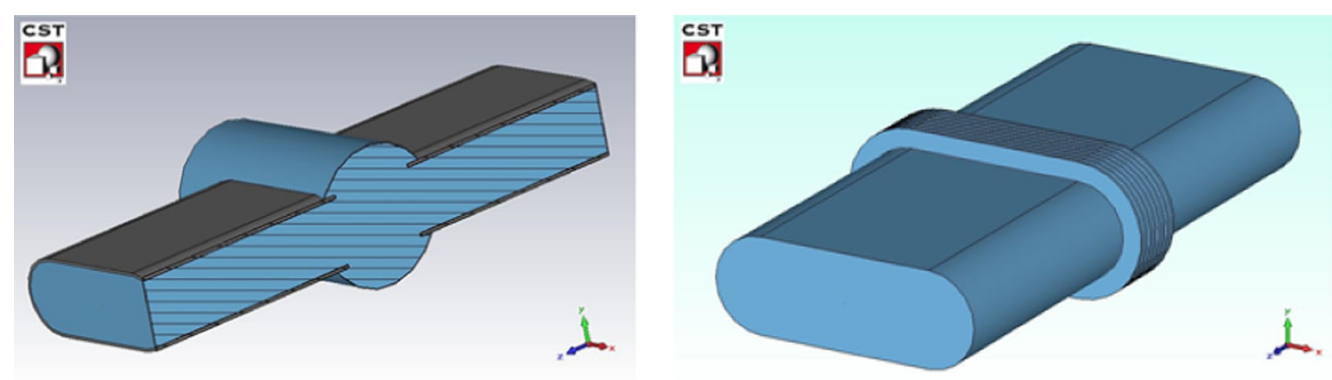

FIG. 8. 3D model of a PS vacuum pump used for CST Particle Studio simulations (left); 3D model of a PS bellow used for CST Particle Studio simulations (right).

Moreover, by summing together all the vertical effective impedances listed in Table VI, we can explain about 10\% of the vertical effective impedance measured at $2 \mathrm{GeV}$, and the $25 \%$ of the vertical effective impedance measured at $25.48 \mathrm{GeV}$.

\section{IMPEDANCE LOCALIZATION MEASUREMENTS}

In order to confirm results from simulation and detect the main sources of impedance in the PS with an experimental approach, impedance localization measurements at the energy of $13.09 \mathrm{GeV}$ were performed. The impedance localization method was proposed at CERN in 1995 [25], when the impedance-induced betatron phase advance shift as a function of intensity was measured in LEP for the first time. Let us consider a transverse impedance source localized in the position $s=s_{k}$ along the accelerator circumference. In case there is no significant cross-talk between adjacent lumped impedances, the effective vertical impedance can be described as

$$
Z_{t}^{\mathrm{eff}}(s) \simeq Z_{t_{k}}^{\mathrm{eff}} L_{k} \delta\left(s-s_{k}\right)
$$

where $L_{k}$ is length of the impedance source in the machine and $\delta(s)$ is the Dirac function. If we substitute Eq. (6) in Eq. (1), we can correlate the tune shift with the intensity to

TABLE VI. Effective horizontal and vertical imaginary part of the effective impedances (sum of dipolar and quadrupolar components) for each type of vacuum equipment, computed from CST Particle Studio simulations. The total impedance is scaled by the total number of elements.

\begin{tabular}{lccc}
\hline \hline & Elements & $Z_{x}^{\text {eff }}[\mathrm{M} \Omega / \mathrm{m}]$ & $Z_{y}^{\text {eff }}[\mathrm{M} \Omega / \mathrm{m}]$ \\
\hline Vacuum pumps & 100 & 0.003 & 0.232 \\
Bellows & 237 & 0.011 & 0.208 \\
Step transitions & 60 & 0.040 & 0.070 \\
Sector valves & 10 & 0.004 & 0.015 \\
Metallic flanges & 44 & 0.004 & 0.022 \\
Total & & 0.052 & 0.55 \\
\hline \hline
\end{tabular}

the imaginary part of the transverse effective impedance for a localized impedance source in the machine:

$$
\frac{\mathrm{d} Q_{t_{k}}}{\mathrm{~d} N_{b}}=-\frac{e^{2}}{8 \pi^{3 / 2} \beta^{2} E_{0} \sigma_{\tau}} \beta_{t}^{0}\left(s_{k}\right) \operatorname{Im}\left[Z_{t_{k}}^{\mathrm{eff}}\right] L_{k}
$$

where $N_{b}$ is the number of particles in the bunch, and $\beta_{t}^{0}(s)$ is the machine unperturbed beta function and $E_{0}=m_{0} \gamma c^{2}$. Considering many impedance sources we have

$$
\frac{\mathrm{d} Q_{t_{\mathrm{tot}}}}{\mathrm{d} N_{b}}=\sum_{k} \frac{\mathrm{d} Q_{t_{k}}}{\mathrm{~d} N_{b}} .
$$

Let us consider now a tune shift provoked by a quadrupolar error $\frac{\mathrm{d} K_{t_{k}}}{\mathrm{~d} N_{b}}$ localized in $s_{k}$. The quadrupolar error shift is related to the tune shift with intensity by

$$
\frac{\mathrm{d} Q_{t_{k}}}{\mathrm{~d} N_{b}}=\frac{\beta_{t}^{0}\left(s_{k}\right)}{4 \pi} \frac{\mathrm{d} K_{t_{k}}}{\mathrm{~d} N_{b}} L_{k}
$$

The variation of the quadrupolar error with intensity is proportional to the imaginary part of a localized transverse impedance by

$$
\frac{\mathrm{d} K_{t_{k}}}{\mathrm{~d} N_{b}}=-\frac{e^{2}}{2 \sqrt{\pi} \beta^{2} E_{0} \sigma_{\tau}} \operatorname{Im}\left[Z_{t_{k}}^{\mathrm{eff}}\right]
$$

A $\beta$-beating and a phase advance beating wave along the machine are associated with the quadrupolar error. In order to calculate these contributions, we define $\mu_{t}(s)$ as the phase advance from the reference BPM located in $s=0$ (reference position) to the $s$ position in the machine, in units of $2 \pi$. The phase advance slope as a function of beam intensity intensity provoked by a quadrupolar error located in $s=s_{k}$ is given by $[26,27]$

$$
\frac{\mathrm{d} \mu_{t}(s)}{\mathrm{d} N_{b}}=\frac{\mathrm{d} Q_{t_{k}}}{\mathrm{~d} N_{b}} \begin{cases}1+\frac{c(s)^{+} s(s)^{+}}{\sin \left(2 \pi Q_{t}^{0}\right)} & \text { for } s \geq s_{k} \\ \frac{s(s)^{-} c(s)^{-}}{\sin \left(2 \pi Q_{t}^{0}\right)} & \text { for } s<s_{k}\end{cases}
$$

where 


$$
\begin{aligned}
& s(s)^{-}=\sin \left(2 \pi \mu_{t}^{0}(s)\right) \\
& c(s)^{+}=\cos \left(4 \pi \mu_{t}^{0}\left(s_{k}\right)-2 \pi \mu_{t}^{0}(s)\right) \\
& s(s)^{+}=\sin \left(2 \pi \mu_{t}^{0}(s)-2 \pi Q_{t}^{0}\right), \\
& c(s)^{-}=\cos \left(2 \pi \mu_{t}^{0}(s)-4 \pi \mu_{t}^{0}\left(s_{k}\right)+2 \pi Q_{t}^{0}\right),
\end{aligned}
$$

and $\mu_{t}^{0}(s)$ is the unperturbed phase advance. The phase advance slope with intensity exhibits a step of $\frac{\mathrm{d} Q_{y_{k}}}{\mathrm{~d} N_{b}}$ at $s=s_{k}$. Considering that the tune shifts related to effective impedance are usually negative, this step will be also negative. It is worth noting that a resistive wall impedance could be treated (on average) as a smoothly distributed quadrupole error provoking a smooth negative slope in the phase advance slope [27].

Measuring the variation of phase advance as a function of intensity along the machine allows us to compute the transverse effective impedance of the devices installed in different locations, and detect the main sources of impedance.

The CERN PS is equipped with 43 multi turn BPMs distributed along the ring, which allow for impedance localization measurements. The localized reactive machine impedance distribution can be measured from the variation of betatron phase advance with intensity between the BPMs. Impedance localization measurements were performed on the vertical plane at the energy of $13.05 \mathrm{GeV}$. A single bunch was injected and accelerated, performing an intensity scan between $6 \times 10^{11}$ and $18 \times 10^{11} \mathrm{ppb}$. An rms bunch length of $14 \mathrm{~ns}$ has been measured with a dedicated tool. We observed a negligible variation during the intensity scan. The beam was excited for about 500 turns by the transverse feedback, tuned at the betatron tune frequency, in order to adiabatically excite the beam natural oscillations. The chromaticity was set as low as possible in order to maintain coherent transverse oscillations for more than 5000 turns, that is the maximum storage limit for the PS BPM system. At each intensity point, the phase advance is measured for each of the machine BPMs with reference to the BPM in section 0 . As a consequence, the phase advance in section 0 is coincident with the machine tune. The phase advance variation with intensity is therefore calculated and the impedance reconstruction method is applied: the response of a thin defocusing lens on the machine phase beating is calculated every $5 \mathrm{~m}$ in the machine. In order to match the measured phase advance slope signal, a matrix of dimensions $126 \times 43$ is built and pseudoinverted applying a least squares minimization algorithm, with the following constraints: 1 . the impedance effect must be defocusing: $\frac{\mathrm{d} K_{y_{k}}}{\mathrm{~d} N_{b}}<0 ; 2$. the sum of all the reconstructed impedances has to match the total transverse impedance computed by the tune shift measurements presented in Sec. III.

The accuracy of the phase advance measurement $\sigma_{\Delta \mu}$ depends on the noise over signal ratio (NSR) in the BPM signal, on the stored number of turns $\mathrm{N}$ and on the Fourier transform method used $F_{\Delta \mu}$ :

$$
\sigma_{\Delta \mu}=F_{\Delta \mu} \frac{\mathrm{NSR}}{\sqrt{N}},
$$

with $F_{\Delta \mu}^{\text {tot }} \simeq 1 / \pi$ for an FFT algorithm.

Considering a set of $M$ phase advance measurements at different intensities $Y_{M}$ with negligible uncertainty in the intensity measurement, assuming the same uncertainty in phase advance for each measurement, as in Eq. (13), the phase advance slope with intensity uncertainty $\sigma_{\Delta \mu / \Delta N_{b}}$ is given by [28]

$$
\sigma_{\Delta \mu / \Delta N_{b}}=F_{\Delta \mu}^{\text {tot }} \frac{\mathrm{NSR}}{\sigma_{Y} \sqrt{M} \sqrt{N}},
$$

where $\sigma_{Y}$ is the standard deviation of the intensity scan $Y$. This uncertainty should be compared with the impedanceinduced phase advance amplitude of Eq. (11):

$$
A_{k}=\frac{\mathrm{d} Q_{t_{k}}}{\mathrm{~d} N_{b}} \frac{1}{\sin \left(2 \pi Q_{t}^{0}\right)} .
$$

It is worth noting that the strength needed to model the resistive wall impedance associated to each reconstructing lens would be too small to be accurate. It is therefore calculated using the PS impedance model and the corresponding impedance-induced phase advance signal is subtracted from the measured one. Figure 9 shows the impedance induced phase advance slope as a function of the BPM locations.

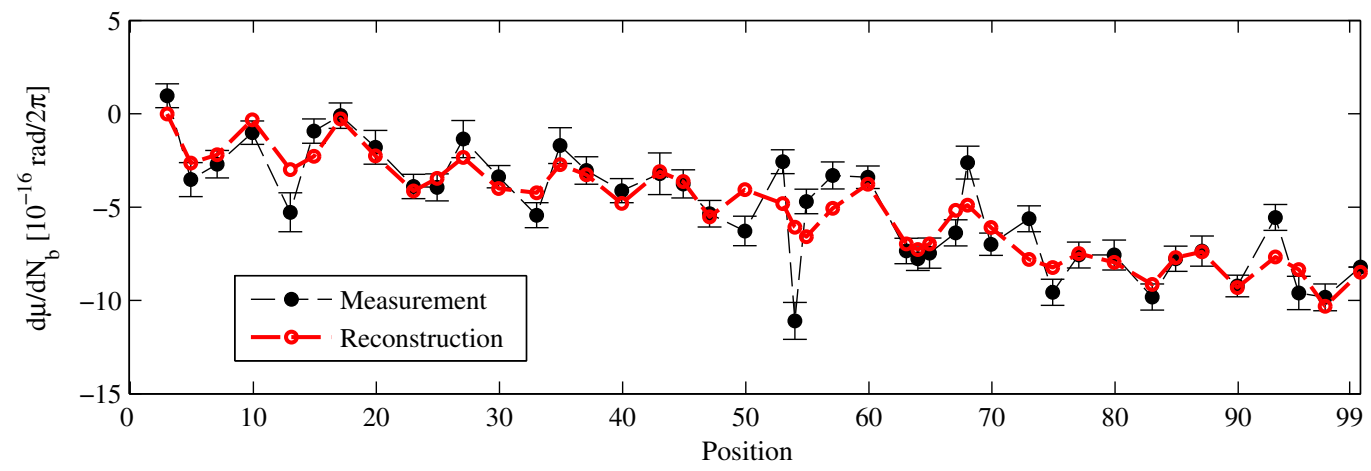

FIG. 9. Comparison between measured and reconstructed phase advance slope as a function of the BPM position along the machine circumference. The horizontal unit represents the straight section number in the PS ring. 


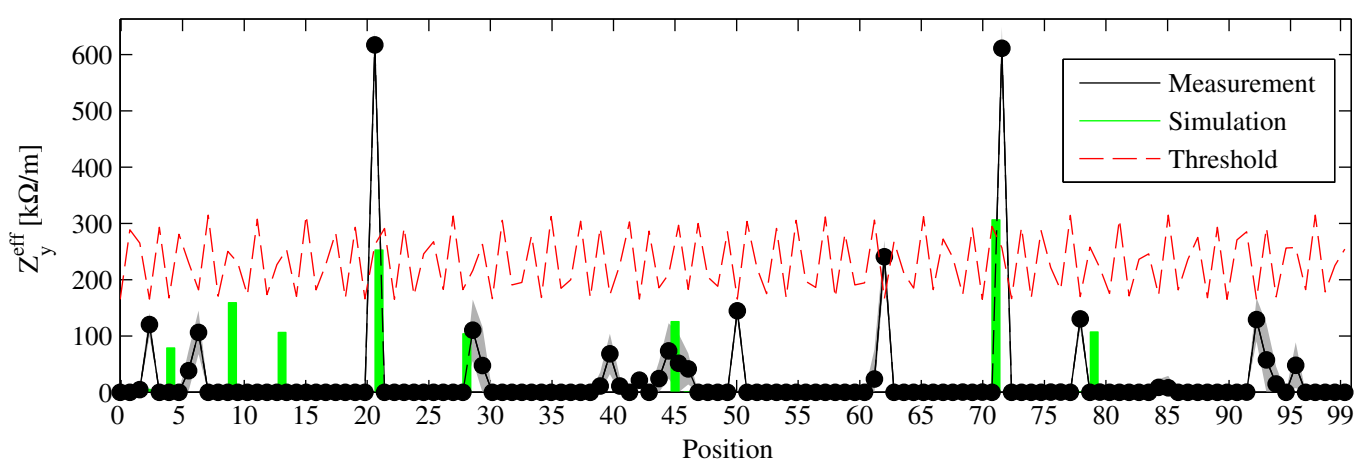

FIG. 10. Comparison between the localized measured and simulated effective vertical impedance, as a function of the impedance reconstruction location along the machine circumference. Considering the noise level in the BPM and the achieved signal amplitude, a threshold for the lowest localizable impedance is deduced.

Figure 10 shows a comparison between the localization measurement and the effective vertical impedance computed with simulations. Considering the noise level in the BPM and the achieved signal amplitude, a threshold for the lowest impedance that can be localized, is deduced. Kickers located in section 21, 28, 45, 71 and 79 have been detected, and the correspondent impedance values are reasonably close, especially for kickers 28,45 and 79 . The other kicker positions could only be approximately detected due to their small impedance. These measurements confirm that the major sources of geometrical impedance in the PS are located in section 71 and in section 21, where three kickers are installed. Measurements at $2 \mathrm{GeV}$ have been described in [27], where analogous conclusions could be drawn, while measurements at $25.48 \mathrm{GeV}$ are planned for the future as an increase in the power of the transverse feedback amplifiers is required.

\section{COMPARISONS BETWEEN MEASUREMENT AND SIMULATIONS}

Figure 11 shows the imaginary part of the effective vertical impedance computed from tune shift

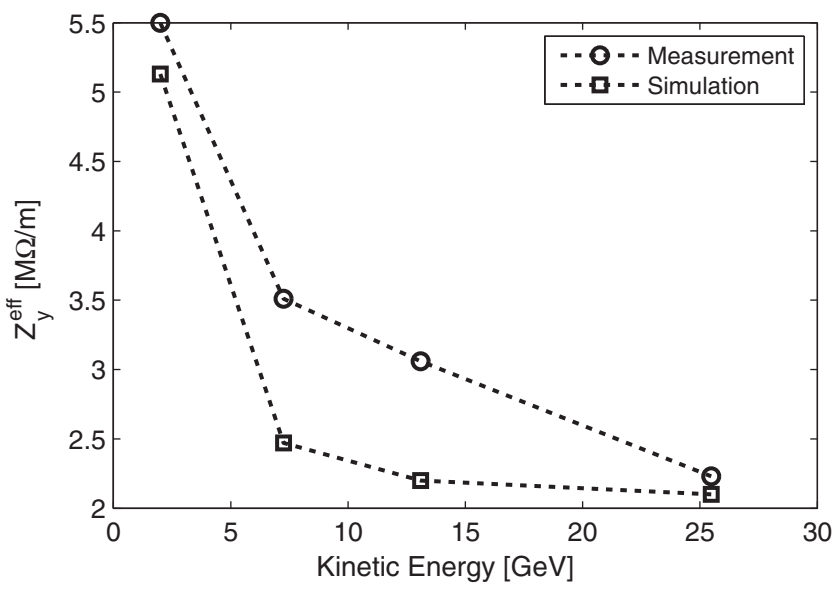

FIG. 11. Imaginary part of the effective vertical impedance computed from tune shift measurements and from the impedance model, as a function of energy. measurements at zero chromaticity, and the imaginary part of the effective vertical impedance computed from simulations, as a function of the energy. At zero chromaticity the measured vertical effective impedance is $5.5 \mathrm{M} \Omega / \mathrm{m}$ at $2 \mathrm{GeV}$ (injection energy) and $2.2 \mathrm{M} \Omega / \mathrm{m}$ at $25.48 \mathrm{GeV}$ (extraction energy), as summarized in Table II. The difference in the two sets of measurements at injection and extraction energies is consistent with the indirect space charge model that we took into account. At injection energy, the space charge contribution is dominant with respect to the contribution of geometrical impedance, as shown in Table VII.

Summing the space charge contribution and the effective impedance due to geometrical impedance introduced by kickers, rf, and vacuum equipment, we obtain a total impedance that explains about $95 \%$ of the measured impedance at 2 and $25.48 \mathrm{GeV}$, and about $70 \%$ of the measured impedance at 7.25 and $13.09 \mathrm{GeV}$. The theoretical total transverse impedance, as a function of frequency, calculated at $2 \mathrm{GeV}$ is shown in Fig. 12. The figure has been obtained by summing the impedances (dipolar and quadrupolar components) of all the machine elements simulated with CST Particle Studio, and the resistive wall and space charge contributions. The impedance model that has been obtained can be used to study transverse beam instabilities in the machine, as the one observed at transition energy [23].

\section{TUNE SHIFT MEASUREMENTS WITH CHROMATICITY SCAN}

According to Eq. (1), the transverse effective impedance does explicitly depend on chromaticity. During tune

TABLE VII. Contribution of the different machine elements to the vertical effective impedance budget $2 \mathrm{GeV}$.

\begin{tabular}{llr}
\hline \hline & $Z_{y}^{\text {eff }}[\mathrm{M} \Omega / \mathrm{m}]$ & Contribution \\
\hline Space charge & 3 & $58.5 \%$ \\
Kickers & 1.4 & $24.9 \%$ \\
Resistive wall & 0.3 & $5.8 \%$ \\
Vacuum & 0.55 & $10.7 \%$ \\
\hline \hline
\end{tabular}


shift measurements at different energies we observed that the chromaticity plays a role in the effective impedance determination. However, our theoretical model predicts a negligible variation of the impedance with chromaticity. To investigate this aspect, several MD sessions have been dedicated to study the dependence of the vertical effective impedance with the vertical chromaticity. Measurements of the tune shift with beam intensity have been acquired at four energies, setting different vertical chromaticities on the machine working point. To change the vertical chromaticity, we need to vary the current in one of the pole face windings, keeping the current of the other three coils and of the figure-of-eight loop to a fixed value. With the use of five independent parameters, we are able to modify only the vertical chromaticity value, keeping constant the horizontal and vertical tunes, horizontal chromaticity, and non linear chromaticity. A measurement of the vertical chromaticity was performed with a dedicated tool after each modification of the working point. The measured value of vertical chromaticity, depending on
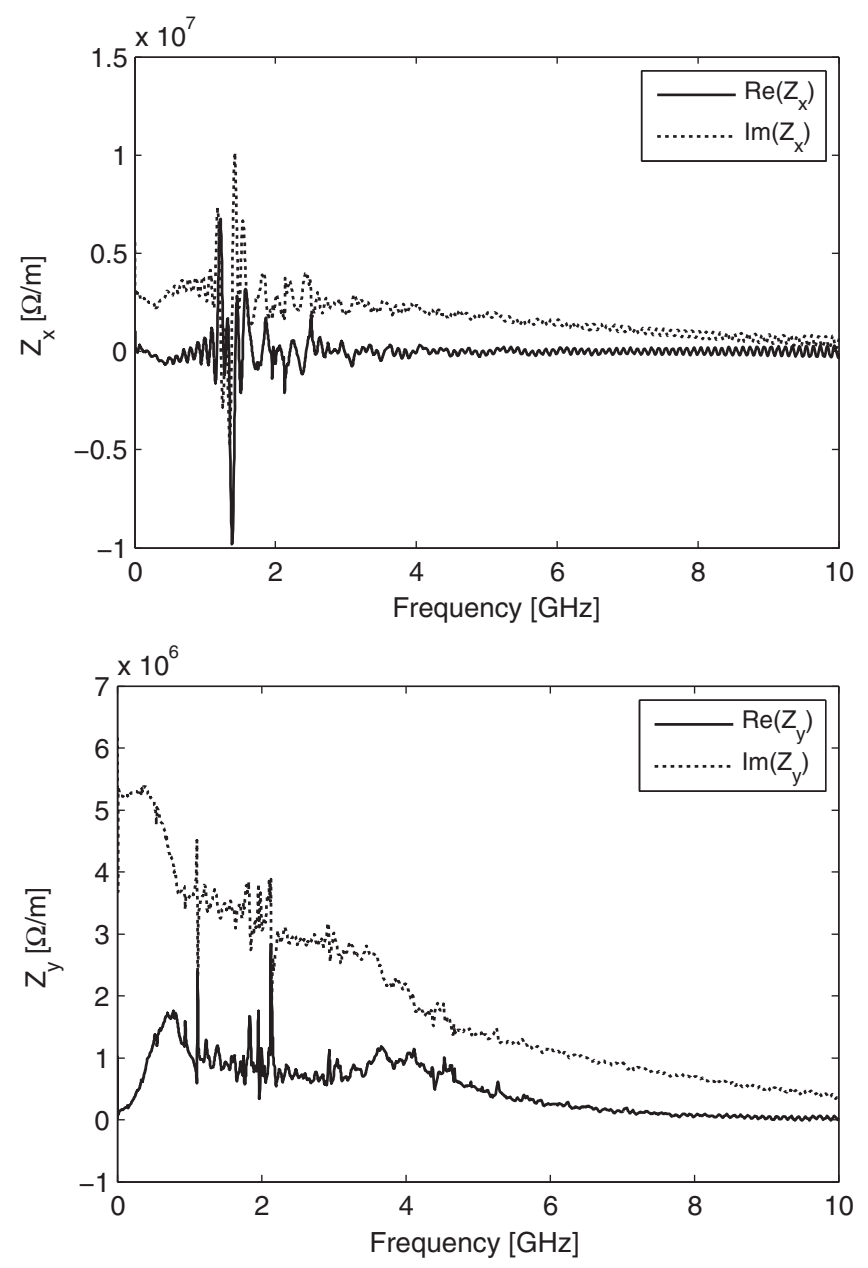

FIG. 12. Horizontal and vertical total PS impedance model (sum of dipolar and quadrupolar components) a $2 \mathrm{GeV}$. The curves are obtained summing the contributions of space charge, resistive wall, kickers, rf, and vacuum equipments. the current that was regulated on the machine coils, may result slightly different from the desired value set on the PS working point application. For instance, setting a vertical chromaticity of -1 in the $2 \mathrm{GeV}$ beam cycle, allows us to measure an effective value of about -1.1 . Four sets of measurements were performed at the kinetic energies of 2, 7.25, 13.09 and $25.48 \mathrm{GeV}$. For each energy, several values of vertical chromaticity have been set and measured. The possibility of pushing the chromaticity to values far from zero, as well as setting a negative chromaticity far below transition energies, was limited by stability of the beam in the specific cycle and by the beam losses in the machine. After each chromaticity measurement, a tune shift scan with intensity was performed in order to measure the effective vertical impedance for the given chromaticity value. The four sets of tune shifts measurements show the same increasing trend of the vertical effective impedance with chromaticity. This behavior could be explained, partially, by the kickers impedance model that has been presented in Sec. IV. In the low frequency vertical impedance of Fig. 6, given by the sum of the vertical impedances of all the PS kickers, we can observe a low frequency resonance, below $1 \mathrm{GHz}$, that can explain, partially, the growth of the impedance with chromatic frequency. We also believe that the presence of metallic inserts in the PS lumped kickers introduce low frequency resonances in the vertical impedance which could further explain the presented measurements. Unfortunately, since we observed some difficulties in processing this type of geometry in CST Particle Studio, we are not able, at this stage, to include this contribution in the model, and we had to account for a simplified geometry of the five lumped kickers. Other possible explanations to validate the presented measurements are still under investigation. In Fig. 13 we show the impedance scan with chromatic frequency at different energies. We can also identify the zero chromaticity impedances presented in the previous section.

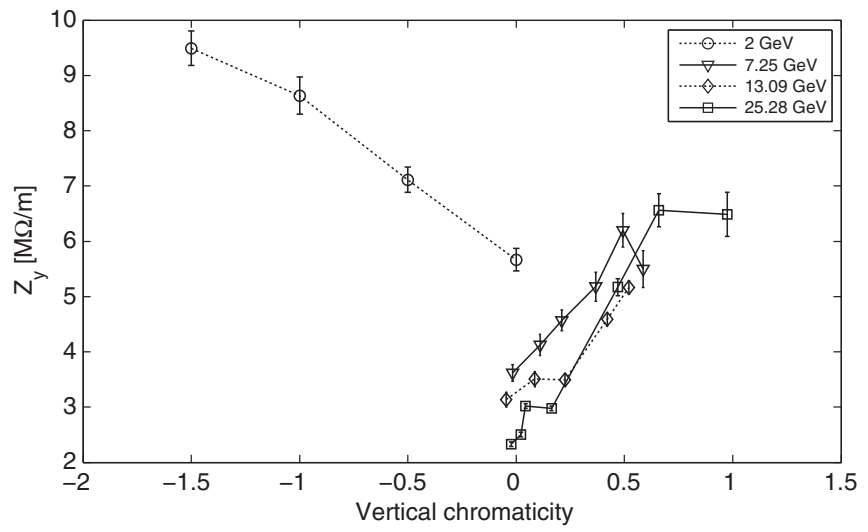

FIG. 13. Imaginary part of the vertical effective impedance as a function of vertical chromaticity at different energies. At injection energy (below transition) the machine operates at negative chromaticity. 


\section{CONCLUSIONS}

In this paper we presented the PS transverse effective impedance obtained at different energies with beam based measurements and numerical simulations. The effective impedance has been computed measuring the betatron tune shift as a function of beam intensity. Measurements have been performed at injection and extraction energies, and at intermediate energies to better understand the evolution of indirect space charge. At zero chromaticity, the measured vertical effective impedance is $5.5 \mathrm{M} \Omega / \mathrm{m}$ at injection energy and $2.2 \mathrm{M} \Omega / \mathrm{m}$ at extraction energy. The difference in the two sets of measurements at injection and extraction energies is consistent with the indirect space charge model that we took into account. The theoretical impedance, evaluated by taking into account the contribution of many machine devices, exhibits an agreement of $70 \%-95 \%$ with the vertical effective impedance computed from tune shift measurements at different energies. These results have also been confirmed with the impedance localization measurements. A scan of the imaginary part of the vertical effective impedance with chromaticity has been performed, revealing an increasing trend with chromaticity for all sets of measurements at different energies. This behavior could be explained, in part, by the kickers impedance model. Other possible explanations to validate the measurements are still under investigation. This impedance model, together with the longitudinal model described in [5], can be used to study collective effects which can play an important role in the LHC injection chain due to the higher beam intensity foreseen within the LHC Injector Upgrade project (LIU). In addition, in the framework of the same project, some important hardware changes will be done in the PS in the next years [29,30,31]. Based on the impedance model presented in this paper, it will be possible to assess their impact on the total transverse effective impedance of the machine.

\section{ACKNOWLEDGMENTS}

We acknowledge for the helpful discussions G. Sterbini, G. Rumolo, V. Vaccaro, R. Wasef, N. Wang, S. Aumon. We thank the PS OP team for the help in setting-up the beams and the CERN impedance team for the useful collaboration.

[1] R. Garoby, Report No. CERNATS-2013-059.

[2] http://hilumilhc.web.cern.ch/.

[3] D. Boussard et al., Mesures d'impedance longitudinale de chambre a vide au PS CERN, 1980.

[4] https://espace.cern.ch/LS1planning/.
[5] M. Migliorati et al., Beam-wall interaction in the CERN Proton Synchrotron for the LHC upgrade, Phys. Rev. ST Accel. Beams 16, 031001 (2013).

[6] R. Klatt, R. D. Kohaupt, and T. Weiland, Theoretical prediction of head tail tune shift in PETRA, in 11th IEEE Particle Accelerator Conference, Vancouver, Canada (1985), pp. 2356.

[7] S. Sacherer, Report No. CERN-PS-BR-76-21, 1976.

[8] R. D. Kohaupt, Transverse Instabilities in PETRA, in 11th Int. Conf. on High-Energy Acc., Geneva, 1980 (1980).

[9] A. W. Chao, Physics of Collective Beam Instabilities in High Energy Accelerators (John Wiley \& Sons, New York, 1993).

[10] M. Gasior, CERN-LHC Project Report 853, 2005.

[11] S. Gilardoni, Fifty years of the CERN Proton Synchrotron (CERN, Geneva, 2011), Vol. 1.

[12] S. Persichelli, Ph.D. thesis, University of Rome La Sapienza, 2015.

[13] C. Zannini et al., Report No. CERN-BE-Note-2010-006, 2009.

[14] K. Yokoya, Resistive wall impedance of beam pipes of general cross section, Part. Accel. 41, 221 (1993).

[15] E. Metral, Report No. CERN-PS-CA 95-24, 1997.

[16] CST AG, Darmstadt, Germany, http://www.cst.com.

[17] H. Chin, Report No. CERN-SL-94-02-AP.

[18] T. Weiland, Transverse beam cavity interaction. Part I: Short range forces, Nucl. Instrum. Methods Phys. Res. 212, 13 (1983).

[19] N. Mounet et al., in Proceedings of the 46th ICFA Advanced Beam Dynamics Workshop on High-Intensity and High-Brightness Hadron Beams HB2010, Morschach, Switzerland, 2010 (PSI, Viligen, Switzerland, 2011).

[20] L. Palumbo et al., IEEE Trans. Nucl. Sci. 31, 1011 (1984).

[21] H. Tsutsui, in Proceedings of the European Particle Accelerator Conference, Vienna, 2000 (EPS, Geneva, 2000).

[22] http://www.3ds.com/products-services/catia.

[23] S. Aumon et al., in Proceedings of the International Particle Accelerator Conference, Kyoto, Japan (ICR, Kyoto, 2010).

[24] R. Losito, Report No. CERN PS/RF Note 96-14.

[25] D. Brandt et al., in Proceedings of the Particle Accelerator Conference, Dallas, TX, 1995 (IEEE, New York, 1995).

[26] R. Miyamoto, Diagnostics of the Fermilab Tevatron Using an AC dipole (VDM Publishing, 2009).

[27] N. Biancacci, Ph.D. thesis, University of Rome La Sapienza, 2014.

[28] R. J. Barlow, A Guide to the Use of Statistical Methods in the Physical Sciences, Manchester Physics Series (Wiley, New York, 1989).

[29] S. Persichelli et al., Impedance studies for the PS Finemet ${ }^{\circledR}$ Loaded longitudinal damper, in Proceedings of IPAC14, Dresden, Germany, 2014 (2014), pp. 1708-1710.

[30] S. Persichelli, Trapped modes in a dummy extraction septum for CERN Proton Synchrotron, Nuovo Cim. C 37, 365 (2014).

[31] S. Persichelli et al., Impedance studies of the dummy septum for CERN PS multi-turn extraction, in Proceedings of IPAC14, Dresden, Germany, 2014 (2014), pp. 1704-1707. 